\title{
A Digital Signal Processing Teaching Methodology Using Concept-Mapping Techniques
}

\author{
M. R. Martínez-Torres, F. J. Barrero García, Senior Member, IEEE, S. L. Toral Marín, Member, IEEE, and \\ S. Gallardo Vázquez
}

\begin{abstract}
The main goal of this study is to develop a scientific method for designing a teaching methodology used in a basic digital signal processing (DSP) course. The proposed method is based on concept-mapping techniques, which applies multivariate statistic analysis to summarize the experience and knowledge of teachers involved in basic DSP teaching. As a result, a set of teaching methodologies is obtained. This result, as well as other information obtained related to the relative importance of the concepts to be covered, has been used to program the course. Moreover, different teaching tools have been developed to implement the proposed teaching methodology. Finally, the reliability of the method has been compared with similar studies to validate the proposed methodology.
\end{abstract}

Index Terms-Computer-based educational tools, concept mapping, digital signal processing (DSP), educational technology, electrical engineering.

\section{INTRODUCTION}

$\mathbf{I}^{2}$ $\mathrm{N}$ recent years, there have been dramatic changes in engineering education, especially in electrical and computer engineering (ECE) education, both in terms of curriculum content (what is taught) and delivery of material (how it is taught) [1]-[5].

On the one hand, emphasis in the electrical engineering field has shifted significantly to the design of digital systems. A shift has been made from emphasizing continuous-time-linear time-invariant systems to discrete-time-linear time-invariant systems. Because of this emphasis on discrete-time systems, the use of digital system processing (DSP), real-time implemented using digital signal processors (DSP devices), is becoming more widespread. The higher educational institution commonly incorporates real-time DSP courses at the sophomore educational level. These courses usually present DSP fundamentals and theory-implementation of discrete-time systems, DSP device structure and programming, finite word length effects, fast Fourier transform (FFT) algorithms, finite impulse response (FIR) and infinite impulse response (IIR) design, multirate DSP, power spectrum estimation, linear prediction, and optimal filtering, etc.-along with a laboratory component that uses evaluation boards, including a DSP device.

On the other hand, technology is everywhere, but does it have a place in the classroom? Since the early days of computers, learning through computer-mediated environments (hypermedia tools, web-based educational support, simulation environments, etc.) has dramatically increased [6]-[8]. However, there are conflicting claims about these learning environments. Some researchers state the validity, even the necessity, of these educational tools, although other authors are more sceptical about them. For instance, a tremendous increase in the use of computer simulation has been noted, sometimes even replacing hardware-based laboratory courses, causing students to miss out on the learning that can only take place in a traditional laboratory. Computer-based educational settings seem to have been implemented without any scientific criteria or, what is worse, without any real necessity. Nevertheless, the pedagogical value of computer-based educational tools has been demonstrated in particular situations. This study demonstrates that the simultaneous integration of traditional and computer-based educational techniques can substantially improve the teaching and learning process.

The need to revise the teaching methodology of engineering has often been discussed. Although many enhancement reformations have been put into practice over the years, not much attention has been paid to scientific analysis for improving the teaching process. The educational process has been evaluated and, at most, empirically improved. This paper presents the application of a scientific method, based on concept-mapping techniques, to design a teaching model of a DSP course, included in the electronic engineering curriculum at the University of Seville, Seville, Spain. The concept-mapping technique has sometimes been used for teaching evaluation [9], but it can also be used for teaching planning [10], as is proposed here. From the concept-mapping analysis, different teaching methods (clusters) have been established verifying many of the improvements that have been recently developed to reform engineering education-active or cooperative learning, technology enhancement, just-in-time learning, and curriculum integration.

This paper is organized as follows. First, the concept-mapping technique is presented in Section II. Then, in Section III, the subject, based on the study of a modern DSP device and its applications, is described, and the concept-mapping technique is used to develop the conceptual framework that guides the educational process. Afterwards, in Section IV, the teaching methodology obtained is analyzed. Finally, the conclusions are drawn.

\section{CONCEPT-MAPPING OVERVIEW}

A concept map is a form of structured conceptualization that can be used by groups to develop the conceptual framework to guide an evaluation, an exercise, a plan, etc. [10], [11]. To 
develop the concept map, a procedure that used quantitative and qualitative features was applied. At the start, the participants generated information through brainstorming. As part of the process, the data was structured, quantified, and analyzed using statistical methods that include a multidimensional scale and clusters analysis. Concept-mapping shows the main categories of mathematically determined ideas derived from the participants' input. Each subset of ideas is represented on the map in cluster form. Those clusters that are closest to each other are said to be more directly linked. The maps represent the opinion of the participants.

The following procedure to develop the concept map is laid out in the following phases [12]:

1) selecting and preparing the participants;

2) brainstorming items (concepts) that deal with the topic matter;

3) structuring and rating those items;

4) representing those items on a concept map (using a multidimensional scale and clusters analysis);

5) interpreting the maps.

\section{APPLICATION TO DSP TEACHING}

The concept-mapping technique has been applied to obtain the teaching methodologies that are best suited for an undergraduate intermediate DSP subject at the University of Seville. This subject focuses on the design of advanced embedded digital systems, DSP devices, and their applications. Special attention is paid to microprocessor architecture and functionality and to practical applications related to digital signal processing algorithms.

The Texas Instruments C3X family has been selected as the DSP device architecture that can fully accomplish the objectives of the subject. The internal structure of C3X family is used to explain complex embedded systems and their utility in DSP area.

The TMS320C3X family is a 32-b floating point, general-purpose, digital signal processor generation from Texas Instruments (TI). ${ }^{1}$ The family architecture features present a central processing module that is common to all family members and has been designed to execute commonly used DSP benchmarks in minimum time for single-multiplier architecture. The internal buses and special DSP instruction set of the TMS320C $3 x$ have the speed and flexibility to execute up to 150 MFLOPS (million floating-point operations per second). It optimizes speed by implementing functions in hardware that other processors implement through software or microcode. This hardware-intensive approach provides performance previously unavailable on a single chip. This DSP can perform parallel multiplications and arithmetic logic unit (ALU) operations on integer or floating-point data in a single cycle. Each processor also possesses a general-purpose register file, a program CACHE, dedicated auxiliary register arithmetic units (ARAUs), internal dual-access memories, one direct memory access (DMA) channel supporting concurrent input-output $(\mathrm{I} / \mathrm{O})$, and a short machine-cycle time. High performance and ease of use are results of these features. General-purpose

${ }^{1}$ The Texas Instruments website is at http://www.ti.com applications are enhanced greatly by the large address space, multiprocessor interface, internally and externally generated wait states, two external interface ports, two timers, serial ports, and a multiple-interrupt structure. Moreover, this environment supports a wide variety of system applications, from host processor to dedicated coprocessor, including high-level language (implemented through its register-based architecture, large address space, powerful addressing modes, flexible instruction set, and well supported, floating-point arithmetic).

The general procedure for concept-mapping development, outlined in the following subsections, is based on the five stages that were described in Section II.

\section{A. Selection and Preparation of the Participants}

One of the most important tasks in developing a concept map is deciding who will participate in the process. Experiments demonstrate that conceptualization is better when the process includes a wide range of expert people. A broad, heterogeneous participation helps to ensure that the different points of view will be considered, thus encouraging "constructing" the right conceptual framework.

For this study, teachers with contrasting work experiences in DSP teaching, particularly with the family TMS320C $3 \mathrm{X}$, have been selected. A total of 14 teachers participated, all of whom had both teaching and DSP application development experience. This number of people is within the adequate limits, between ten and 20 [10].

\section{B. Brainstorming}

The next stage consisted of establishing a list of items related to the teaching of the proposed course. Using the brainstorming technique, the work group identified a list of 82 items, shown in Table I.

\section{Structuring and Rating the Items}

When a set of items that describes the conceptual domain of the given topic is established, one must provide information about how they are interrelated and measured in relation to the topic. Both tasks make up the stage of item structuring throughout the concept-mapping development process.

The first task of the working group consisted of classifying the 82 items in several groups based on their affinity with respect to some common teaching methodology. Each of the participants applies his or her personal experience to define the number of groups and the teaching methodology. A similarity matrix, defined as $S_{n \times n}$ with $n=82$, is obtained as follows: the value of the $(i, j)$ element is equal to 1 if the $i$ th and the $j$ th items are grouped together and is equal to 0 otherwise. The total similarity matrix, defined as $T_{n \times n}$, is obtained as the addition of all the similarity matrixes.

The second task involved rating each item according to its contribution to the goals of the course. The items then had to be entered on the scoring table in Likert scale form, with a range of 1 to 5 , considering that $1=$ "little contribution," $5=$ "a lot of contribution," and the numbers in between referred to intermediate contributions. A "zero-contribution" score was not possible, since the brainstorming stage specifically asked for those 
TABLE I

LIST OF IDENTIFIED ITEMS

\begin{tabular}{|c|c|}
\hline Serial port (SP) operation & TMS320C3x architecture \\
\hline SP: configuration \& control registers & Microprocessor and microcontroller versus DSP device \\
\hline DSK30 overview & Computer architecture and technology \\
\hline DSK30-PC communication & Low power modes in TMS320C3x: description \\
\hline Harvard architecture: TMS320C3x. & Solution in designing a microprocessor system: discussion \\
\hline Branch with delay & Timers: low frequency clock generation \\
\hline Stack in a microprocessor & SIM3x \\
\hline SP programming & Parallel instructions \\
\hline $\begin{array}{l}\text { Low power modes in TMS320C3x: CPU } \\
\text { waking up }\end{array}$ & Microprocessor technological evolution \\
\hline $\begin{array}{l}\text { TMS320C3x family: C30, C31, C32 and } \\
\text { VC33 }\end{array}$ & Interrupt vectors, considerations in microcomputer mode \\
\hline Pipeline conflicts & External peripheral interface \\
\hline Reset and interrupt vectors & Boot-loader \\
\hline $\begin{array}{l}\text { DSP device special instructions and } \\
\text { addressing modes }\end{array}$ & Wait state generation \\
\hline AIC (TLC32040) configuration & RDY signal \\
\hline MCBL versus MP modes & External access timing \\
\hline Addressing modes & DSP device applications \\
\hline DMA: internal structure & Interrupts: context switching \\
\hline DMA: internal registers & DSP devices: manufacturers \\
\hline External access using TMS320C3x & Programming the timers \\
\hline Data management in TMS320C3x & Repeat mode \\
\hline Bit-reversed addressing mode & Programming the DMA \\
\hline Address space & Using the DMA: An example \\
\hline $\begin{array}{l}\text { Working methodology in DSP devices } \\
\text { based projects }\end{array}$ & CACHE memories: general description \\
\hline Circular indirect addressing mode & CACHE memory in TMS320C3x \\
\hline Internal bus operation: pipelining & Boot-loader using SP: an example \\
\hline AIC (TLC32040) overview & DSP application: a digital wave generator \\
\hline Interlock instructions & Machine cycle in TMS320C3x \\
\hline $\begin{array}{l}\text { Design of digital systems based on } \\
\text { TMS320C3x }\end{array}$ & Description of DSP devices-based real projects \\
\hline CPU internal registers & Integer versus floating point format \\
\hline $\begin{array}{l}\text { Timing diagrams in read or write } \\
\text { operations }\end{array}$ & Assembler programming using DSK30 \\
\hline Direct addressing mode & Interrupt generation in SIM3x \\
\hline Micro-operations in TMS320C3x & Using the debugger and the DSK30 \\
\hline Timers: internal structure & Circular addressing mode: an example \\
\hline Timers: internal registers & $\begin{array}{l}\text { CPU-peripheral interface using data, control, and } \\
\text { addressing buses }\end{array}$ \\
\hline RDY generation & Description of a microprocessor system components \\
\hline $\begin{array}{l}\text { DSP devices in commercial world (their } \\
\text { utility in a real electronic engineering } \\
\text { design company) }\end{array}$ & $\begin{array}{l}\text { Design of a digital system using a TMS } 320 \mathrm{C} 3 \mathrm{x} \text { and some } \\
\text { functional specifications }\end{array}$ \\
\hline Von-Neumann versus Harvard & Instruction set \\
\hline Interconnecting DSP devices & Final cost of a digital design based on a TMS320C3x \\
\hline Interrupt vector table & Superscalar versus VLIW \\
\hline $\begin{array}{l}\text { Implementing digital filtering using the } \\
\text { DSK30 }\end{array}$ & Computer architecture evolution \\
\hline Memory map & Peripherals using the same memory addressing space \\
\hline
\end{tabular}

items that contributed to obtaining the course goals. Therefore, to a greater or lesser extent, all the items had some contribution.

\section{Representing the Items in a Concept-Map Form}

Furthermore, a double analysis was performed using information about the following statistical analysis: a multidimensional scale and a cluster analysis. To complete this double analysis, MATLAB was used.

Multidimensional scaling is a mathematical tool that uses proximities between objects, subjects, or stimuli to produce a spatial representation of these items. The proximities are defined as any set of numbers that expresses the amount of similarity or dissimilarity between pairs of objects, subjects, or stimuli.
The object of a multidimensional scale is to find the coordinates of the points in $p$-dimensional space so that there is a solid agreement between the observed proximities and the interpoint distances [13]. In concept mapping, this multidimensional scale sets up a point map that represents the set of declarations made during the brainstorming session. It is based on the results of the similarity matrix of the classified task. The most common approach used to determine the coordinates of the objects is an iterative process, commonly referred to as the Shepard-Kruskal algorithm [13].

The multidimensional scale gives the analyst a specific number $p$ of dimensions that represent the set of points. If a one-dimensional (1-D) solution is required, all the points will form one line. A two-dimensional (2-D) solution will place the 


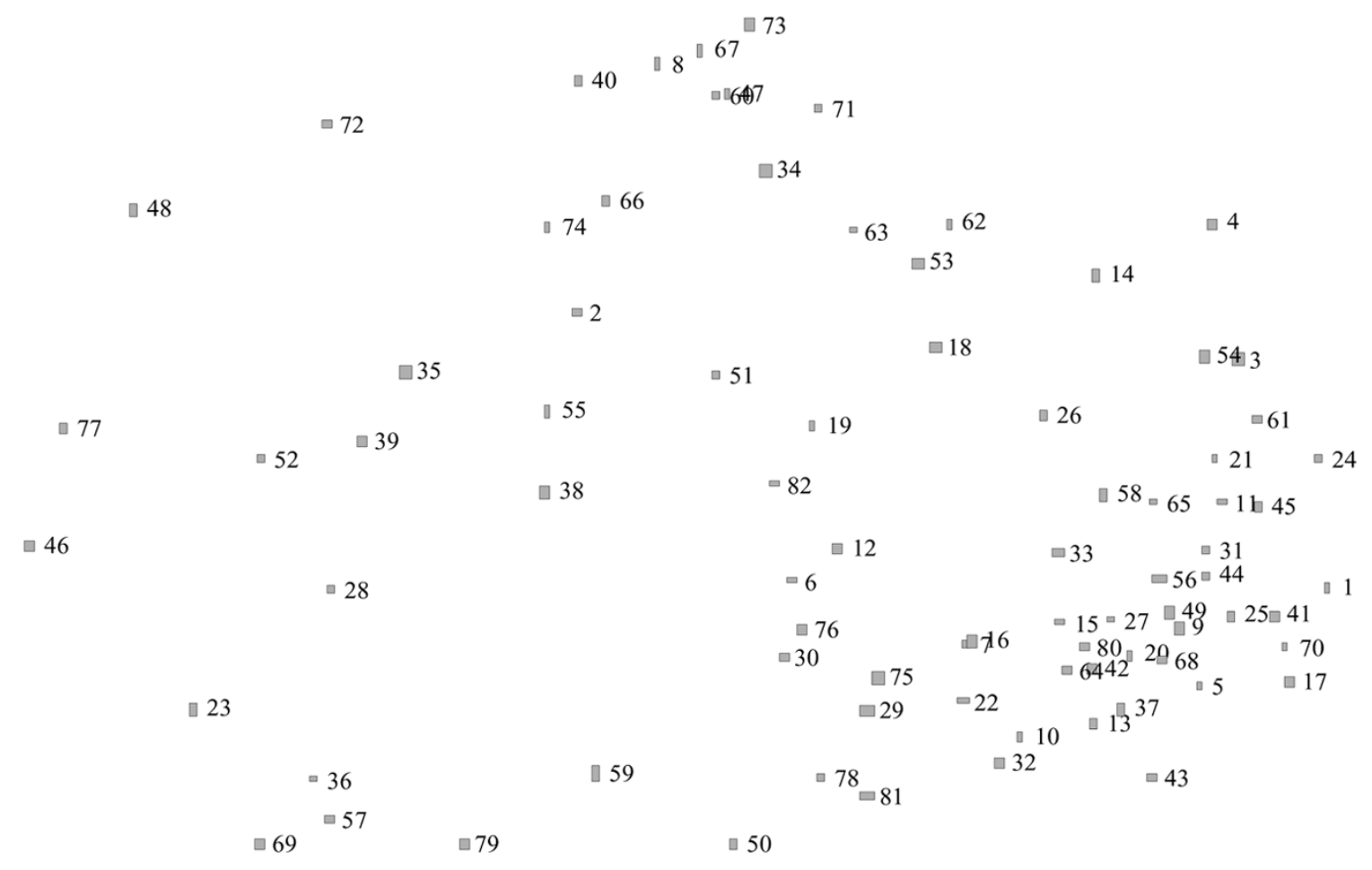

Fig. 1. Point map obtained from multidimensional scaling.

set of points on a plane. The analyst would be able to use these dimensions. On the other hand, interpretation of solutions with more than three dimensions is difficult. Therefore, when using concept maps, one should use 2-D graphs.

In this case, the different items were distributed on a 2-D plane, starting from the similarity matrix, in such a way that the distance between the different items is inversely proportional to their affinity. That is, those items situated closer to each other are conceptually more closely related than those that are placed further from the plane. By representing the information in a 2-D space, the loss of information to obtain a less complicated interpretation of the information is accepted.

The cluster analysis organizes the information coming from the multidimensional scale, not from the similarity matrix [14]. Ward's algorithm was used for the cluster analysis since it offers more sensitive solutions and can be better interpreted than other estimates [15].

At first, the cluster analysis considers each item as its own cluster, thus obtaining a solution of $N$ clusters, in this case 82 , corresponding to the number of identified items. For each level of analysis, Ward's algorithm combines two clusters until finally all the items are combined into just one cluster. Determination of the number of clusters to be used in the final solution is important. Subsequently, discretion is required when examining the different types of possible cluster solutions to decide which ones make sense. As a rule use of the number of clusters that errs by excess, rather than by defect, is normal; in other words, a larger number of clusters is preferred to having a cluster containing heterogeneous concepts.

Once the multidimensional scale and the cluster analysis are carried out, a point map and a cluster map are formed. The final analysis requires an average score for each participant for each item and for each cluster, forming a point-rating map and a cluster-rating map.

\section{E. Interpretation of Maps}

In order to interpret the maps, a final workgroup was organized. Generally, the results derived from the cluster analysis are more difficult to interpret than those from the multidimensional scale. The cluster analysis is seen as an indicator. At times, one would like to "visually arrange" the clusters into sensitive parts so that the multidimensional space could be interpreted more easily. The key is to maintain the integrity of the multidimensional scale results by achieving a solution that will not allow the clusters to overlap. A consensus of the names given to the different clusters must be reached, using as a starting point those names given to the groups by the participants.

\section{CONCEPT-MAPPING APPLICATION RESULTS}

The point map of Fig. 1 is the result of multidimensional scaling. Each number represents an item of Table I. The distance between points is a measure of affinity. Closer items are more closely related to each other, while further points show a high level of dissimilarity. The resultant map is a (bidimensional) approach of the distances obtained from the sum of each participant similarity matrix.

Fig. 2 illustrates the rating point map, derived from the value that the participants assigned to each item, following the Likert 1-to-5 scale. In the upper left-hand corner of the figure, the correspondence between layers and numerical Likert values are shown.

Once the bidimensional representation of the 82 items is achieved, the items must be grouped in homogeneous clusters. These clusters will define the resulting teaching methodologies. 


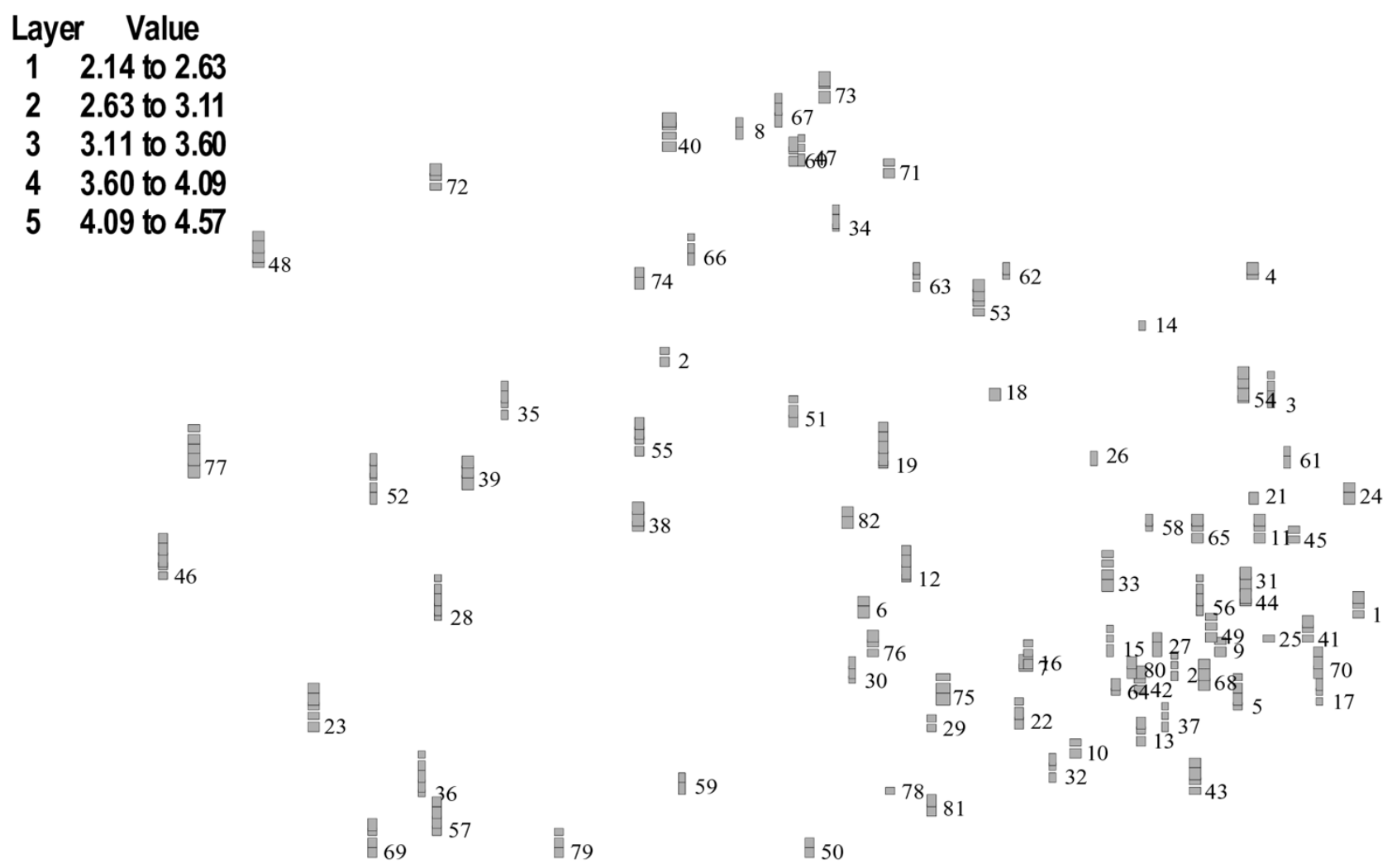

Fig. 2. Rating point map obtained from multidimensional scaling.

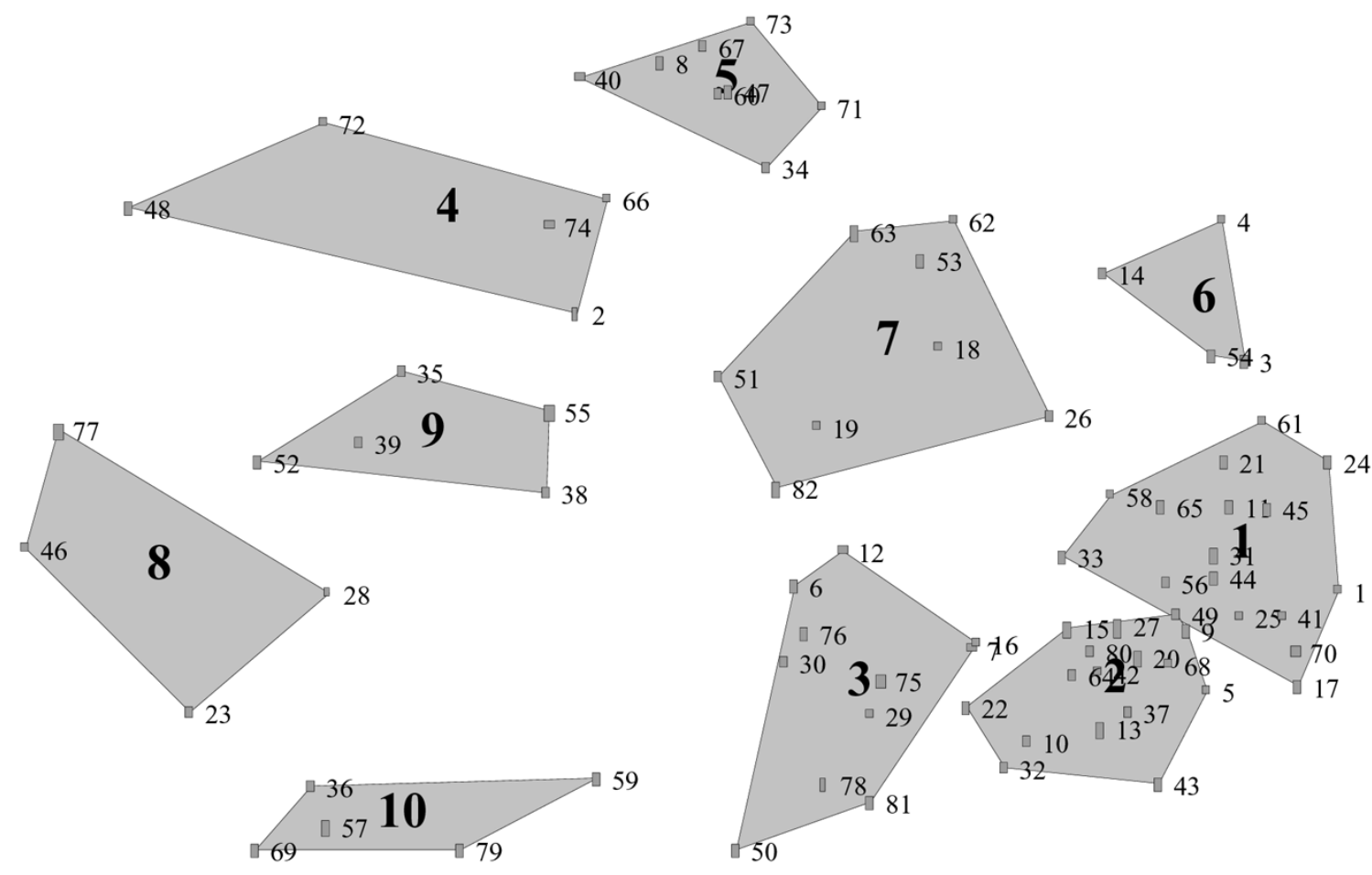

Fig. 3. Cluster map.

The result of the cluster analysis is summarized in Fig. 3. According to Wards' algorithm, the items are grouped in ten clusters. The concurring denominations of the ten clusters are shown in Table II.
Three groups can be observed in the cluster map (Table II). The first one is based on clusters numbers 1, 2, 3, 6, and 7. It includes cluster number 2 , which implies a conventional lecture. Clusters numbers 3 and 7 (theoretical and practical self-learning 

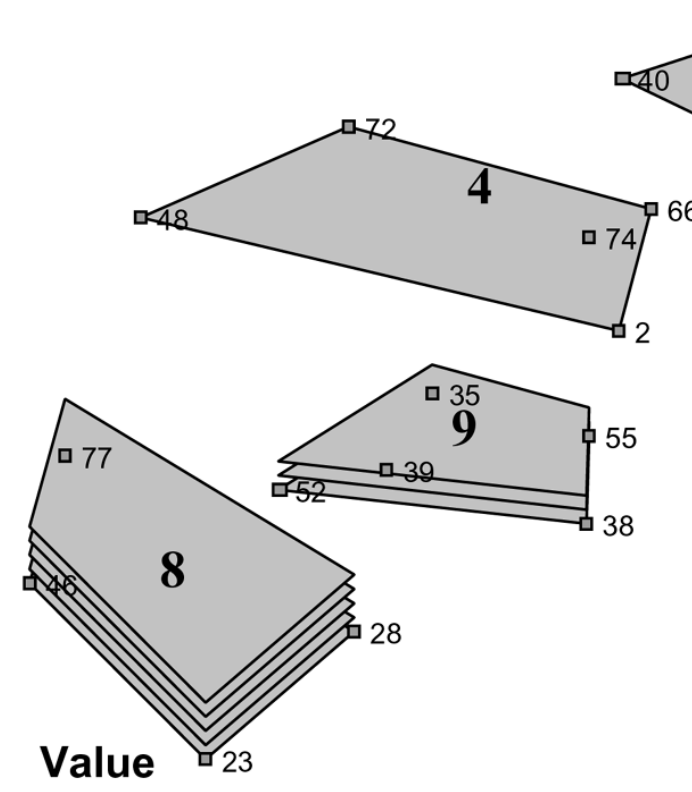

Layer Value 23

$\begin{array}{ll}1 & 3.13 \text { to } 3.36 \\ 2 & 3.36 \text { to } 3.59 \\ 3 & 3.59 \text { to } 3.82 \\ 4 & 3.82 \text { to } 4.05 \\ 5 & 4.05 \text { to } 4.29\end{array}$

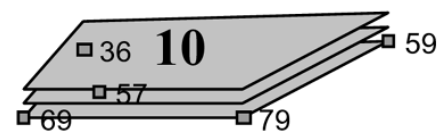

59

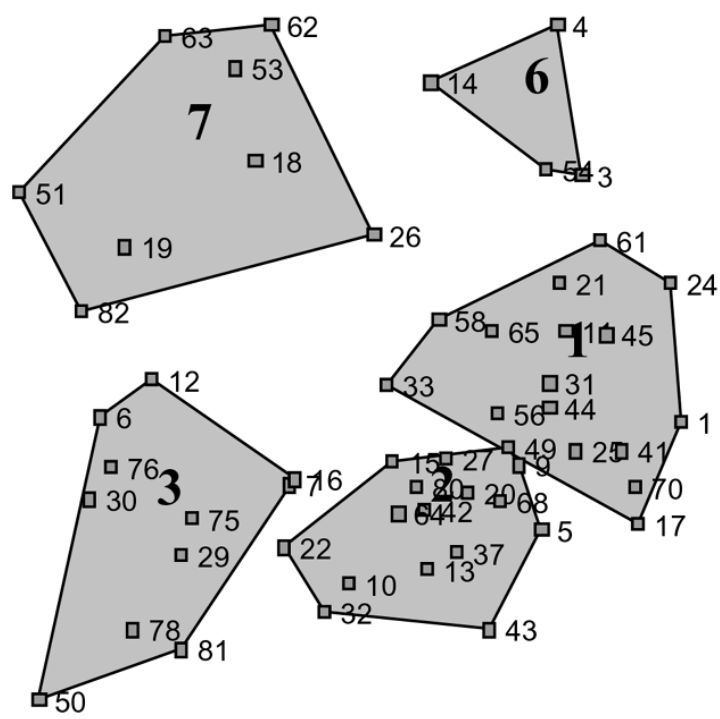

Fig. 4. Cluster rating map.

TABLE II

Cluster ANALysis Results

\begin{tabular}{cl}
\hline \hline Cluster & \multicolumn{1}{c}{ Denomination } \\
\hline $\mathbf{1}$ & Orientated learning using hypermedia tools \\
$\mathbf{2}$ & Conventional lecture \\
$\mathbf{3}$ & Theoretical self-learning with reference material \\
$\mathbf{4}$ & Lab classes using a simulating tool \\
$\mathbf{5}$ & Lab classes using a debugger and a designer starter kit \\
$\mathbf{6}$ & Seminar about the use of practical tools: simulator, board ... \\
$\mathbf{7}$ & Practical self-learning with reference material \\
$\mathbf{8}$ & Conferences, discussions \\
$\mathbf{9}$ & Practical applications and problems solved in classes \\
$\mathbf{1 0}$ & Real world contact: visiting EE companies \\
\hline \hline
\end{tabular}

using reference material) appear very close to the second one. In these cases, the learning process calls for the active participation of students with adequate reference material. Cluster 1 also belongs to this group. In this particular case, an oriented or guided learning using multimedia teaching tools has been defined. This teaching methodology considers the active participation of students in their learning process, in accordance with the future European Credits Transfers System (ECTS), [16], [17]. Nonetheless, the teaching process is not just reduced to conventional classes. For instance, cluster 1 explicitly shows those items for which one must be trained using novel computerbased teaching tools, such as multimedia animations. Under this cluster, items such as DSP special addressing modes, internal bus operations, pipelining operations, and conflicts or CACHE memory, are found. These concepts are very difficult to explain using conventional classes. However, any multimedia tool, including animations to show the associated dynamic data flow, is specially indicated for the teaching process. Finally, cluster 6 ties theory and laboratory work in the subject together by means of laboratory seminars on software and hardware tools to be used throughout the course.

The second group is based on clusters 4,5 , and 9, related to laboratory work. In this case, a simulation tool and a designer kit are used in laboratory classes to develop practical applications. This cluster relates to the practical point of view of the theoretical concepts described in cluster 1 .

The last group embodies clusters number 8 and 10. It connects the subject to the real and professional world. To meet this goal, talks, conferences, and debates (cluster 8) and visits to firms working in the DSP and microelectronic design areas (cluster 10) are proposed. The principal aim is to bring the company perspective closer to the students: making decisions, looking for solutions, developing analysis skills, contacting the working environment, employing real applications, etc.

Fig. 4 depicts the cluster rating map. The rating equivalence is shown in the lower left-hand corner. Clusters number 8, 9, and 10 are predominant versus the others which have a similar weight. To summarize, clusters related to practical applications and offering external activities are rated as predominant.

\section{A. Reliability Analysis}

The traditional theory normally applied to the reliability of social science research does not correctly fit on concept maps, since the assumption is that for each item tested there is a correct answer known in advance. Therefore, each individual is measured for the question and marked only as correct or incorrect. Later, the reliability for each test item(s) or for the total score is estimated. However, with concept maps, one answer is not assumed to be correct or incorrect. To measure reliability, the data 
TABLE III

DESCRIPTIVE STATISTICS FOR RELIABILITY ESTIMATES FOR 38 CONCEPT MAPPING PROJECTS

\begin{tabular}{ccccccc}
\hline \hline & $\mathbf{R}_{\mathbf{I I}}$ & $\mathbf{r}_{\mathbf{I T}}$ & $\mathbf{r}_{\mathbf{I M}}$ & $\mathbf{r}_{\mathbf{R R}}$ & $\mathbf{r}_{\text {SHT }}$ & $\mathbf{r}_{\text {SHM }}$ \\
\hline Number of projects & 33 & 33 & 33 & 37 & 33 & 33 \\
Mean & 0.815 & 0.929 & 0.863 & 0.783 & 0.833 & 0.551 \\
Median & 0.820 & 0.930 & 0.862 & 0.821 & 0.848 & 0.558 \\
Minimum & 0.670 & 0.882 & 0.740 & 0.427 & 0.724 & 0.259 \\
Maximum & 0.934 & 0.973 & 0.954 & 0.935 & 0.932 & 0.907 \\
SD & 0.070 & 0.022 & 0.047 & 0.121 & 0.054 & 0.155 \\
\hline \hline
\end{tabular}

matrix structure is inverted (with respect to traditional theory) so that the persons are placed in columns, and the items (or pairs of items) are placed in rows. The value of reliability focuses on consistency via the group of supposedly homogeneous participants. In that respect, talking about the reliability of the similarity matrix or the reliability of the map, but not the reliability of the individual statements, is helpful [18].

The key product of the concept mapping process is the 2-D map itself; consequently, the efforts made to verify the reliability are directed toward the central phases of analysis, development, and representation.

In the study published by Trochim [18], the reliability of concept mapping was tested by six coefficients that could be easily estimated from the available data on any concept map project. These coefficients were defined and estimated for 38 concept map projects. The results indicated that the concept-mapping process could be considered reliable, according to the standards generally recognized for acceptable reliability levels. This study only considered the concept-mapping reliability.

On these concept maps, a test to determine whether the reliability levels were within the acceptable reliability standards was conducted.

All of the reliability estimators referred to on the concept maps and used in Trochim's reliability study were calculated for this study, and they were compared with the results obtained in Trochim's research (Table III). A high level of reliability was found within these concept maps. Furthermore, the indicators were found to be valid and within the standards shown by Trochim. The coefficients defined by Trochim are as follows.

1) Individual-to-Individual Sort Reliability $\left(\mathbf{r}_{\mathbf{I I}}\right)$ : Correlates each person's binary sort matrix $S_{n \times n}$ for each pair of individuals; it explains how the sorts are correlated for the different participants in the development of the concept map. This correlation is identified by calculating the average of the correlations and by applying the Spearman-Brown Prophecy formula [19], as follows:

$$
r_{k k}=\frac{k \bar{r}_{i j}}{1+(k-1) \bar{r}_{i j}}
$$

where

$r_{i j} \quad$ correlation estimated from the data;

$k=N / n$ where $N$ is the total sample size, and $n$ is the sample size on which $r_{i j}$ is based;

$r_{k k} \quad$ reliability estimated according to the SpearmenBrown Prophecy formula.

In this case, the reliability was shown to be 0.703 , a value that is less than Trochim's average values, but still found to be within the limits; the minimum value obtained by Trochim was
0.670, as shown in Table III. Therefore, this value is considered acceptable.

2) Individual-to-Total Matrix Reliability $\left(\mathbf{r}_{\mathbf{I T}}\right)$ : This correlates each person's binary sort matrix $S_{n \times n}$, with the total matrix $T_{n \times n}$; it determines how the sorts carried out by each person correlate with all the sorts. To find the calculation, one must take the average of these correlations and apply the Spearmen-Brown Prophecy formula. The value of the individual to total matrix reliability is 0.918 . This value is close to the average value, which indicates that the reliability of this type is valid on this map.

3) Individual-to-Map Reliability $\left(\mathbf{r}_{\mathrm{IM}}\right)$ : This correlates each person's binary sort matrix $S_{n \times n}$, with the Euclidean matrix distances $D_{n \times n}$. To find this calculation, one takes the average of these correlations and applies the Spearmen-Brown Prophecy formula. In this case, the value was 0.882 . This value is higher than the average value found in Trochim's research.

4) Average Intersort Reliability $\left(\mathbf{r}_{\mathbf{R R}}\right)$ : This calculates the correlation among the scores of each pair of persons. To find the calculation, one takes the average of these correlations and applies the Spearmen-Brown Prophecy formula. This concept map showed the value was 0.745 , which demonstrates that the scores found on this concept map were relatively reliable since this value was close to the average of Trochim's concept map.

5) Split-HalfReliabilities $\left(\mathbf{r}_{\mathbf{S H T}}\right.$ and $\left.\mathbf{r}_{\mathrm{SHM}}\right)$ : Divide the set of sorts from each project into two halves and calculate concept maps for each group. The total matrices $T_{A}$ and $T_{B}$ are correlated, and then the Spearmen-Brown Prophecy formula is applied to obtain $r_{\mathrm{SHT}}$. The Euclidean distances were correlated between all pairs of points on the two maps $D_{A}$ and $D_{B}$, and the Spearmen-Brown correction was applied to achieve $r_{\mathrm{SHM}}$. The similarity matrix result $\left(r_{\mathrm{SHT}}\right)$ and the distance matrix result $\left(r_{\mathrm{SHM}}\right)$ were close to the average values defined by Trochim. Therefore, these values are considered acceptable.

In short, this concept map is reliable since the different reliability indicators showed values within the acceptable limits set by Trochim.

\section{CONClusion}

A scientific method for designing a teaching methodology has been used for planning a basic digital signal processing (DSP) course. The proposed method, based on concept-mapping techniques, has applied multivariate statistic analysis to summarize the experience and knowledge of teachers involved in the educational process. Consistently, a set of ten teaching methodologies has been obtained for programming the course. The teaching 
methodology has also been analyzed by calculating the reliability estimators. Finally, it has been validated by comparing the obtained reliability estimators within the Trochim's limits.

\section{REFERENCES}

[1] R. M. Felder, G. N. Felder, and J. Dietz, "A longitudinal study of engineering students performance and retention v. comparisons with traditionally-taught students," J. Eng. Educ., vol. 98, no. 4, pp. 469-480, 1998.

[2] L. Carley, P. Khosla, and R. Unetich, "Teaching introduction to electrical and computer engineering in context," Proc. IEEE, vol. 88, pp. 8-22, Jan. 2000.

[3] J. Wilson and W. Jennings, "Studio courses: How information technology is changing the way we teach, on campus and off," Proc. IEEE, vol. 88, pp. 72-80, Jan. 2000.

[4] T. Roppel, "An interdisciplinary laboratory sequence in electrical and computer engineering: Curriculum design and assessment results," IEEE Trans. Educ., vol. 43, no. 2, pp. 143-152, May 2000.

[5] R. L. Taylor, D. Heer, and T. S. Fiez, "Using an integrated platform for learning to reinvent engineering education," IEEE Trans. Educ., vol. 46, no. 4, pp. 409-419, Nov. 2003.

[6] S. Bagui, "Reasons for increased learning using multimedia," J. Educational Multimedia Hypermedia, vol. 7, pp. 3-18, 1998.

[7] P. Aedo et al., "Assessing the utility of an interactive electronic book for learning the pascal language," IEEE Trans. Educ., vol. 43, no. 3, pp. 403-413, Aug. 2000.

[8] W. Christian and M. Belloni, Teaching physics with interactive curricular material. Englewood Cliffs, NJ: Prentice-Hall, 2001.

[9] W. M. K. Trochim, "Criteria for evaluating graduate programs in evaluation," Evaluation News Comment: The Mag. Australasian Evaluation Soc., vol. 5, no. 2, pp. 54-57, 1996.

[10] - "An introduction to concept mapping for planning and evaluation," Evaluation Program Planning, vol. 12, no. 1, pp. 1-16, 1989.

[11] J. F. Vega-Riveros, G. P. Marciales, and M. Martínez, "Concept maps in engineering education: A case study," Global J. Eng. Educ., vol. 2, no. 1, pp. 21-27, 1998.

[12] D. G. Kolb and D. M. Shepherd, "Concept mapping organizational cultures," J. Manag. Inquiry, vol. 6, no. 4, pp. 282-295, 1997.

[13] L. Fahrmeir and A. Hamerle, Multivariate Statistische Verfahren. Berlin, Germany: De Gruyter, 1984.

[14] B. S. Everitt, Cluster Analysis. New York: Halsted, 1993.

[15] J. H. Ward, "Hierarchical grouping to optimize an objective function," J. Amer. Statistical Assn., vol. 58, pp. 236-244, 1963.

[16] European Commission, "Realising the European higher education area," in Communiqué Conf. Ministers Responsible for Higher Education, Berlin, Germany, Sep. 19, 2003.
[17] European Commission, "The European higher education area," in Joint Declaration Eur. Ministers of Education Bologna, Italy, Jun. 19, 1999.

[18] W. M. K. Trochim, "The reliability of concept mapping," presented at the Annu. Conf. Amer. Evaluation Assn., Dallas, TX, Nov. 6, 1993.

[19] J. C. Nunnally, Psychometric Theory, 2nd ed. New York: McGrawHill, 1978

M. R. Martínez Torres was born in Madrid, Spain, in 1973. She received the Business Administration and Ph.D. degrees from the University of Seville, Seville, Spain, in 1996 and 2003, respectively.

She is currently a Full Professor in the Business Administration and Marketing Department, University of Seville. Her research interests include intellectual capital and knowledge management.

F. J. Barrero García (M'03-SM'05) was born in Seville, Spain, in 1967. $\mathrm{He}$ received the electrical engineering and $\mathrm{Ph} . \mathrm{D}$. degrees from the University of Seville, Seville, Spain, in 1992 and 1998, respectively.

In 1992, he joined the Department of Electronic Engineering at the University of Seville, where he is currently a Full Professor. His current interests include microprocessor and digital signal processing device systems, fuzzy-logic-based system, control of electrical drives, and power electronics.

S. L. Toral Marín (M'01) was born in Rabat, Morocco, in 1972. He received the electrical engineering and Ph.D. degrees from the University of Seville, Seville, Spain, in 1995 and 1999, respectively.

He is currently a Full Professor in the Electronic Engineering Department, University of Seville. His research interests include microprocessor and digital signal processing device systems, stochastic processing, and their industrial applications.

S. Gallardo Vázquez was born in Huelva, Spain, in 1978. He received the telecommunication engineering degree from the University of Seville, Seville, Spain, in 2002. He is currently working toward the Ph.D. degree in electronic engineering, signal processing, and communications at the University of Seville, Seville, Spain.

In 2003, he joined the Department of Electronic Engineering at the University of Seville as a Researcher on a research project aimed at power electronic conversion control strategies. His research interests include digital signal processing device systems, information and communication technologies, and power electronics. 